\title{
IMAGE CURRENTS IN AZIMUTHALLY INHOMOGENEOUS METALLIC BEAM PIPES
}

\author{
F. Caspers, A. Mostacci*, F. Ruggiero, CERN, Geneve, Switzerland \\ L. Palumbo, Università di Roma "La Sapienza", Roma, Italy
}

\begin{abstract}
We consider an ultra-relativistic particle travelling onaxis in an infinitely long cylindrical metallic beam pipe with azimuthally varying conductivity. A semi-analytical solution, obtained by applying approximate boundary conditions, predicts an image current distribution on the pipe walls practically independent of the azimuth, at least in the frequency range relevant for future machines such as the LHC. We discuss numerical simulations and bench measurements which confirm the theoretical predictions. Implications for the beam-induced ohmic losses in the copper coated, welded LHC beam screen are also addressed.
\end{abstract}

\section{INTRODUCTION AND MOTIVATIONS}

The distribution of the image currents generated by a particle beam travelling in metallic beam pipes whose conductivity varies with the azimuth (but is constant in the $z$-direction), is important in order to quantify the beamwall interaction in that case. If the currents would avoid the badly conducting region (flowing mainly in the good conductor), the beam would practically not "see" it. On the contrary, if the image currents are constant over the azimuth (TEM-like field distribution), i.e. if the same amount of current flows in the good as in the bad conductor, then the ohmic losses may be driven by the bad conducting part, even if it is smaller than the good conductor.

This problem is of primary interest in the design of the LHC liner where the copper coating (high conductivity) is interrupted by the welding strip which is much smaller (a factor 1/60 in azimuth ) but which can have a much lower conductivity (roughly up to a factor $10^{-3}$ ). The impact of such a strip on the total heating power has to be estimated.

The fields generated by a single ultra-relativistic particle travelling on-axis in an infinitely long, azimuthally inhomogeneous, metallic beam pipe can be found semianalytically under some simplifying assumptions. The main ones are a circular cross section, a conductivity varying sinusoidally with the azimuth, a wall thickness greater than the skin-depth (at any frequency of interest) and the use of Standard Impedance Boundary Conditions (SIBCs) [1]. The semi-analytical solution is then simply derived by magnetic and electric Hertzian potentials whose amplitudes depend on the driving term (the charge field) and on the boundary conditions.

Such a solution [2] tends to a DC-like behaviour (image currents avoiding the bad conducting part) only for extremely low frequencies (typically below $1 \mathrm{~Hz}$ ). On the contrary, in the practically relevant part of the spectrum, the

\footnotetext{
* Also with Università di Roma "La Sapienza", Roma, Italy
}

magnetic field on the boundary (i.e. the image currents) is found to be independent of the azimuth. This is consistent with a first order approach, which assumes on the boundary the same magnetic field as for a charge in the free space.

To asses these results, numerical simulations and bench measurements have been performed independently. The conclusions are reported in Sec. 2 and Sec. 3 respectively. Lastly Sec. 4 discusses implications in the estimate of the heating power dissipated in the LHC liner.

\section{NUMERICAL RESULTS}

There is an analogy between the effects of a given structure on a particle beam and the properties of the coaxial transmission-line obtained by inserting a metallic wire on the axis of the structure itself. This analogy, first developed in 1974 [3], is now widely used in a number of different situations and the coaxial wire method is one of the most common bench measurements to estimate the energy lost by a particle going through an accelerator component. The coaxial wire method can also be easily implemented on a general purpose electromagnetic fields simulator such as the High Frequency Structure Simulator (HFSS) [4].

In our case, the simulated structure is simply a cylindrical pipe (circular cross section) with a metallic wire on its axis; the longitudinal length is equal to twice the wavelength $\lambda(\lambda=30 \mathrm{~cm})$. The radius of this model is $4 \mathrm{~cm}$ (about a factor 2 bigger than the LHC liner), while the radius of the wire is $2 \mathrm{~mm}$. Only the TEM wave is propagating in the structure since the cut-off frequency of the $T E_{11}$ mode for this model is roughly $2 \mathrm{GHz}$. The power flowing into the coaxial transmission line is $1 \mathrm{Watt}$ and only one mode is included in the ports solution. For numerical convergence reasons also HFSS assumes (by default) the SIBCs to avoid solving for the field inside the conductors.

Taking a $90^{\circ}$ slice (with appropriate symmetry boundary conditions) of a pipe made of stainless steel and a perfect conductor (using copper the result doesn't change), we get for the magnetic field on the boundary the pattern shown in Fig. 1 (upper plot). The lower plot compares $H_{\phi}$ to $H_{z}$, both on the boundary. The fields are normalised by the azimuthal magnetic field $H_{\phi}^{I}$ in a coaxial line fed with the same power.

The magnetic field is azimuthal $\left(H_{\phi} \gg H_{z}\right)$ and constant along the azimuth as predicted from the theory. The maximum value of the normalised azimuthal magnetic field should be unity; the small difference observed can be explained by the uncertainty on the value of the radius of the wire, which is so small that it is comparable with the size of the meshes close to it. 

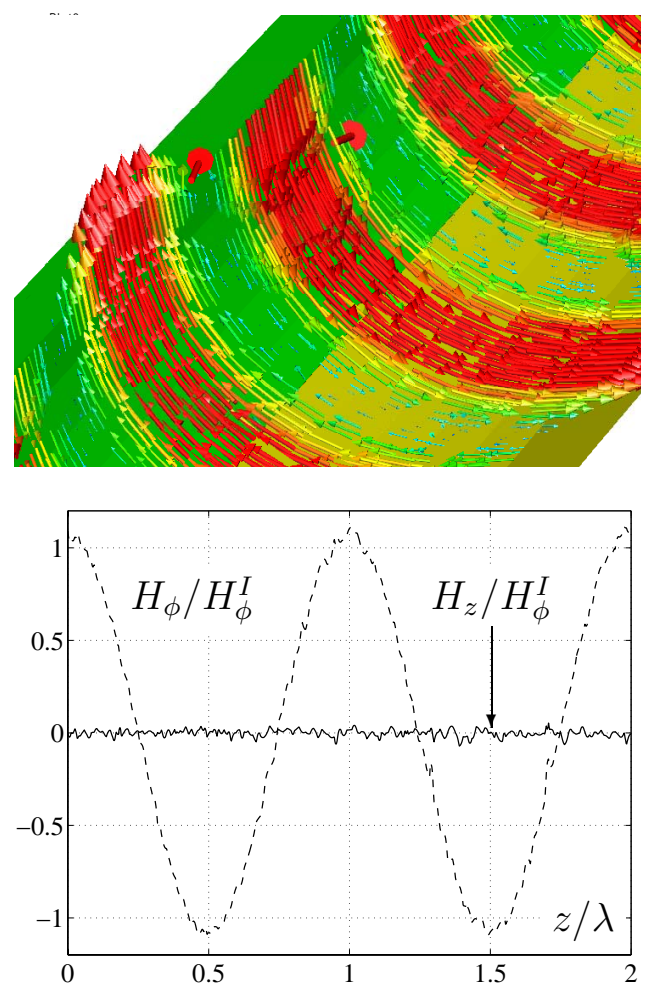

Figure 1: Magnetic field on the boundary (upper plot) and $H_{\phi}$ (lower plot, dashed line) and $H_{z}$ (lower plot, solid line) along the $z$-axis and normalised to the field in a perfect coaxial line fed with the same power. The green part is a perfect conductor while the yellow one is stainless steel (50000 tetrahedrons are used).

\section{EXPERIMENTAL RESULTS}

To assess the theoretical and numerical predictions, an experimental study has been performed on a bench prototype. A cylindrical pipe (of radius $2.5 \mathrm{~cm}$ ) made of 12 different bars which can be mounted and dismounted with a reasonable mechanical precision, has been designed and built. Each bar is "identical" to the others in shape, so that they can be interchanged; they are kept in place by external rings with screws. There are four such rings, since the entire structure is $70 \mathrm{~cm}$ long (see Fig. 2). The bars are available in two sets, one set of 12 steel bars (bad conductor) and one set of 12 brass bars (good conductor). The prototype can be mounted in all possible combinations, from all brass bars to all steel bars.

To make precise, reliable and meaningful measurements, the pipe has been transformed into a TEM resonator by inserting a cylindrical tube (silver-plated stainless steel) along the axis of the structure; the inner cylinder is kept in the right position by Teflon supports (see Fig. 2, lower right photo). The diameter of the silver-plated tube is $1 \mathrm{~cm}$ and its length is $50 \mathrm{~cm}$; it is chosen to be of stainless steel to ensure mechanical stability and it is silver plated to reduce ohmic losses. The coupling circuit is also shown in Fig. 2 (lower left photo); the little disk on the edge of the

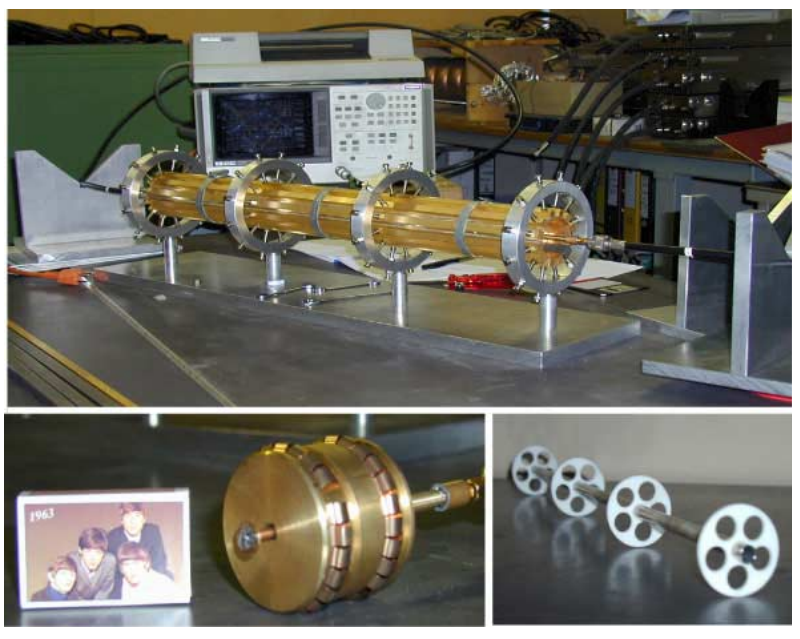

Figure 2: $Q$-factor measurements set-up.

electrode increases the coupling capacity, giving stability to the resonance frequency with respect to the positioning of the coupling electrode.

If the azimuthal magnetic field along the pipe circumference is constant, the power loss inside our cavity will be simply the sum of the power dissipated in the inner conductor $\left(P_{\text {inner }}\right)$ and the power dissipated in each of the 12 bars making up the pipe. Then exchanging a good conducting bar (brass bar) with a steel bar will increase the losses in the resonator (since the current on the wall flows along a more resistive path) and a decrease of the $Q$-factor should be observed. The $Q$-factor is measured in transmission with a network analyser HP8753D.

A number of measurements has been performed replacing brass bars with steel ones. In the following, when referring to $Q$-factors we will refer to the $Q$-factor of the TEM resonator which can be obtained correcting the $Q$ measured with the instrument from the effect of the coupling circuit. In order to distinguish between different set-ups we introduce a suffix indicating the number of steel bars mounted; for instance $Q_{N}$ refers to the prototype when $N$ steel bars and $(12-N)$ brass bars are mounted $\left(Q_{0}\right.$ refers to the situation with only brass bars). Typical measurements concern $Q_{0}, Q_{1}, Q_{2}, Q_{3}$. Since the steel bars are found to be remarkably different (geometrically and in their surface properties), they have been labelled ("bar 1", "bar 2", "bar 3") and we refer to the $Q$-factor measured with all brass except the steel "bar $j$ " $(j=1, \ldots, 3)$ as $Q_{1}^{j}$.

It can be shown from the definition of the $Q$-factor, that if the power dissipated in one bar does not depend on the other bars making up the pipe (i.e. the same current flows in each bar), the following relation holds

$$
\frac{Q_{0}-Q_{N}}{Q_{N}} \approx \sum_{j=1}^{N} \frac{Q_{0}-Q_{1}^{j}}{Q_{1}^{j}} .
$$

Such an equation is appealing because it is independent of the values of $P_{\text {inner }}$ and even of the electrical properties 
of the single "bar $j$ ", provided that $Q_{1}^{j}$ and $Q_{N}$ are measured $^{1}$. The drawback is that we have to make at least four measurements $\left(Q_{0}, Q_{2}, Q_{1}^{1}, Q_{1}^{2}\right)$ and then compare them. The result is likely to be affected by the mechanical tolerances which enter whenever we dismount a bar to substitute it with another one, and they are mainly responsible for our measurement uncertainty.

Labelling the LHS and RHS of Eq. (1) as $f$ and $g$ respectively, Table 1 compares the estimated values $\bar{f}$ and $\bar{g}$ over a set of at least 3 independent measurements and their uncertainty $u_{c}(f)$ and $u_{c}(g)$, defined according to [5]. The measurements were performed at two resonating frequencies of the structure (namely $288 \mathrm{MHz}$ and $1.16 \mathrm{GHz}$ ).

Table 1: Analysis of the measured data. To confirm the theoretical predictions, numerical values in the first and in the third columns should be identical (bold values). The uncertainty for each value is also reported (cf. text).

\begin{tabular}{|c|c|c|c||c|c|}
\hline & & $\bar{f}$ & $u_{c}(f)$ & $\bar{g}$ & $u_{c}(g)$ \\
\hline \multirow{2}{*}{$\begin{array}{c}\text { steel } \\
\text { bars }\end{array}$} & $288 \mathrm{MHz}$ & $\mathbf{0 . 7 0 4}$ & 0.02 & $\mathbf{0 . 6 8 7}$ & 0.03 \\
\cline { 2 - 6 } & $1.16 \mathrm{GHz}$ & $\mathbf{0 . 5 8 9}$ & 0.01 & $\mathbf{0 . 5 7 9}$ & 0.02 \\
\hline \hline \multirow{2}{*}{$\begin{array}{c}\text { s steel } \\
\text { bars }\end{array}$} & $288 \mathrm{MHz}$ & $\mathbf{1 . 0 3 7}$ & 0.02 & $\mathbf{1 . 0 4 1}$ & 0.03 \\
\cline { 2 - 6 } & $1.16 \mathrm{GHz}$ & $\mathbf{0 . 8 7 7}$ & 0.01 & $\mathbf{0 . 8 8 0}$ & 0.02 \\
\hline
\end{tabular}

The results are in good agreement with the theoretical expectations. The bold values in Table 1 representing the LHS and RHS of the identity (1) are very close and their relative uncertainty is smaller than $5 \%$. In conclusion, the theoretical result of a constant magnetic field on the metallic pipe is confirmed within the measurement uncertainty.

\section{HEATING POWER IN THE LHC LINER}

A welding in the copper-coated LHC beam screen can be considered as a small strip of a bad conductor (approximately stainless steel), covering about $1 / 60$ th of the entire LHC liner cross section.

Since the same current flows both in the copper and in the bad conductor in the liner at any relevant frequency, we can apply the perturbative approach (which was only postulated before), in the same way as shown in [6] (but considering the real cross section and not only its circular approximation). The beam screen, in fact, has roughly a circular shape flattened on two opposite sides (the width is $44 \mathrm{~mm}$ and the height is $36 \mathrm{~mm}$ ). The following numerical values assume an average beam current of $536 \mathrm{~mA}$ and the r.m.s. bunch length of $7.5 \mathrm{~cm}$. The equivalent effective DCconductivity of copper measured in liner samples varies

\footnotetext{
${ }^{1}$ This is very relevant for the steel bars because the steel has also magnetic properties $\left(\mu_{r} \neq 0\right)$ which cannot be easily estimated at the measurement frequency. In fact in our set-up, we used (for convenience) the kind of steel used in civil construction, and its magnetic properties are not reported in the data-sheets.
}

from $4.6 \times 10^{7} \mathrm{~S} / \mathrm{m}$ at room temperature to $1.3 \times 10^{9} \mathrm{~S} / \mathrm{m}$ at LHC operating conditions (liquid He temperature and high magnetic field) [6], while the welding conductivity is assumed close to the stainless steel one $\left(2 \times 10^{6} \mathrm{~S} / \mathrm{m}\right)$ always.

The small welding strip will dissipate about $7 \%$ of the power dissipated in the copper coated part at room temperature. At cryogenic temperatures, the effect is more pronounced (since the conductivity of the copper increases but the electric properties of the welding remain nearly unchanged) and the small strip will dissipate about $34 \%$ of the power dissipated in the copper. In this last case the total expected heating power is roughly $105 \mathrm{~mW} / \mathrm{m}$ (considering the values of the conductivities reported above). The previous numbers refer to the real liner cross-section: the effect of the non circular shape has been estimated comparing the solution of the Poisson equation in the real cross section and in the circumscribed one, as previously done in [7] for the older liner proposal. The effect is found to be marginal. The actual heating power is very close to what is reported in [6], but that estimate assumed a circular pipe with a radius of $17.4 \mathrm{~mm}$ and an average beam current of $560 \mathrm{~mA}$ [8] without including the effect of the welding strip.

\section{CONCLUSIONS}

We have presented numerical simulations and bench measurements to check the distribution of image currents generated by an ultra-relativistic beam travelling on-axis in an azimuthally inhomogeneous metallic beam pipe. The azimuthal magnetic field on the boundary is constant with the azimuth at any practically relevant frequency and thus the same image current flows both in the better conducting region as well as in the worse one. This implies that in the LHC liner the image current will not avoid the welding strip and the parasitic heating power is about $105 \mathrm{~mW} / \mathrm{m}$ at LHC operating conditions (35\% more than in the case of a beam screen made only of copper).

\section{REFERENCES}

[1] T.B.A. Senior, J.L. Volakis, "Approximate boundary conditions in electromagnetics", IEE, London, 1995.

[2] A. Mostacci, "Beam-wall interaction in the LHC liner", $\mathrm{PhD}$ thesis, 2001 (unpublished).

[3] M. Sands, J. Rees, SLAC-Report PEP-95, August 1974.

[4] See World Wide Web address, www . ansoft.com.

[5] International Organization for Standardization et al., "Guide to expression of uncertainty in measurement", ISO, Geneva, 1995.

[6] F. Caspers, M. Morvillo, F. Ruggiero, J. Tan and H. Tsutsui, EPAC 2000, Vienna, August 2000 (pp. 376-378).

[7] F. Ruggiero, CERN SL/95-09 (AP) and Particle Accelerators, 50, 1995.

[8] J. Tan, private communication, May 2001. 